observatoires convenablement répartis en longitude, afin d'éviter les lacunes dues au mauvais temps.

Elle attire l'attention sur la nécessité de diffuser ces ursigrammes quotidiens suivant le même code, à des heures voisines, mais ne se recouvrant pas.

\title{
Commission 12. (RAdiation et SPECTROscopie solaires)
}

I. La Commission considère que la publication d'une table révisée du spectre solaire dans le plus court délai possible est de la plus haute importance pour les astronomes qui s'occupent de recherches sur le soleil et sur les spectres stellaires. Pour réduire le délai de publication, la Commission I2 recommande que la table révisée soit limitée à l'intervalle spectral encore inédit de $\lambda 3063$ à $\lambda 6600$, et que les longueurs d'onde soient données dans l'échelle de 1928. A cet effet, la Commission recommande que l'U.A.I. accorde une subvention de $£ 500$ ou de $\$ 2000$ pour la publication de la table solaire révisée.

\section{Commission i6. (OBservations Physiques des Planètes)}

I. La Commission décide de ne donner des nouveaux noms qu'à des formations lunaires importantes parmi celles qui n'ont pas encore été désignées.

Elle propose de donner le nom de Deslandres à la grande dépression rectangulaire située au sud du mur droit et qui contient les cratères Hell et Lexell.

2. La Commission recommande aux observateurs de la Lune de travailler uniquement sur des gabarits tracés au moyen de photographies à très grande échelle prises dans des conditions d'éclairement appropriées.

Elle recommande aux sections lunaires des associations astronomiques de s'entendre directement entre elles et avec les observatoires bien équipés pour l'obtention de telles photographies.

3. La Commission recommande aux observateurs qui étudient les colorations des planètes, d'utiliser des dispositifs corrigeant la dispersion atmosphérique, lorsque les observations sont faites à une faible hauteur.

\section{Commission I7. (Mouvement et Figure de LA LUNe)}

I. La Commission est unanime à estimer qu'il serait important d'entreprendre la réduction de l'ensemble des observations relatives à la libration de la lune par une méthode rigoureuse. L'exécution de cette grande tâche serait facilitée par la création d'un laboratoire mathématique international, qui serait une section de l'Observatoire International projeté.

2. La Commission est unanime à estimer que les travaux poursuivis par M. C. B. Watts (Washington) sur les irrégularités du limbe lunaire sont importants et méritent tout l'appui de la Commission.

3. La Commission est unanime à donner acte au 'Nautical Almanac Office' de ce que les prédictions d'occultations telles qu'elles sont calculées par ses soins sont fort utiles et contribuent au progrès de la branche de l'Astronomie dont s'occupe la Commission.

\section{Commission I8. (LongItUdes PAR TÉLÉGRAPHIE SANS FIL)}

I. Comme suite aux décisions prises lors de l'Assemblée Générale de Stockholm en I938, la Commission demande à l'Assemblée Générale que la publication déjà entreprise des Résultats de l’Opération mondiale des Longitudes de I933 soit liquidée aux moindres frais dans les conditions qui sont précisées dans le Rapport de son Président (voir 'Draft Reports', pp. 87-90) et qui correspondent à un partage exact des dépenses avec l'Association Internationale de Géodésie. 
Elle demande que la somme de 800 dollars prévue dans ce Rapport soit mise par le Comité Exécutif à la disposition du Bureau Central de l'Association Internationale de Géodésie, qui assurera la publication et la diffusion des résultats finaux et des conclusions.

2. La Commission I8 (Longitudes par Télégraphie sans Fil) a décidé de demander à l'Assemblée Générale de l'Union sa transformation en une Commission de détermination des positions géographiques.

A cette extension sensible de ses attributions actuelles devra correspondre une composition de ses membres également plus étendue, pour laquelle la Commission s'en remet au Comité Exécutif de l'Union.

\section{Commission r9. (VARIATION DES LATITUDES)}

I. La Commission tient à exprimer sa sincère gratitude pour le travail de Monsieur Carnera, en particulier pendant la période difficile que nous avons traversée.

2. La Commission recommande que le Bureau Central soit transféré de Capodimonte, où M. Carnera le dirigeait, à Turin, où M. Cecchini se chargera de la direction.

3. La Commission sollicite la coopération du Gouvernement du 'Commonwealth of Australia', afin d'obtenir que les observations de variation de latitude soient continuées à Adelaide, à cause de leur grande valeur pour le programme international d'observations concertées. Une aide financière pour la construction d'un bâtiment et l'exécution du travail pour une période provisoire de 7 ans, assurerait que ce travail important soit repris.

4. La Commission demande une subvention spéciale de huit mille frans-or pour la publication des résultats des observations de variation de latitude jusqu'à la fin de cette année.

5. La Commission recommande que la subvention annuelle de trois mille francs-or, précédemment allouée au Bureau Central, soit renouvelée.

6. La Commission demande une subvention spéciale de mille francs-or pour couvrir les frais de déménagement des archives, documents, machines à calculer, etc. du Bureau Central de Naples à Turin.

7. Il est instamment recommandé que les cahiers d'observation soient transmis par les stations de latitude au Bureau Central aussi rapidement que possible, afin que le Bureau Central puisse exécuter son travail et en publier les résultats assez tôt pour satisfaire les besoins des usagers.

8. Il est proposé que l'U.A.I. exprime à l'Instituto para a Alta Cultura et à l'Observatoire astronomique de Lisbonne le grand intérêt qu'elle porte à l'exécution d'observations de variation de la latitude au moyen du grand télescope zénithal Askania, appartenant à l'Instituto Geografica e Cadastral de Lisbonne.

9. L'attention est appelée sur le grand intérêt qu'il y aurait à établir des lunettes photographiques zénithales en différents points de la terre, non seulement pour améliorer nos connaissances sur le mouvement du pôle, mais aussi pour receuillir les données indispensables relativement aux mouvements propres en déclinaison, ainsi que des données utiles pour la détermination des constantes du système solaire. Pour déterminer le mouvement du pôle, il faudrait disposer de plusieurs stations de même latitude, tandis que pour déterminer les mouvements propres des stations de latitudes différentes seraient nécessaires.

\section{Commission 20. (Petites Planètes, Comètes et Satellites)}

I. La Commission recommande que désormais la comète périodique Pons-CoggiaWinnecke-Forbes soit dénommée comète Crommelin.

2. La Commission recommande que l'adjonction de nombres au noms des comètes soit seulement pratiquée dans le but de faire une distinction entre les comètes périodiques 